\title{
PARAMETRIZED BORSUK-ULAM THEOREMS FOR MULTIVALUED MAPS
}

\author{
MAREK IZYDOREK AND JAN JAWOROWSKI
}

(Communicated by Frederick R. Cohen)

\begin{abstract}
By combining parametrized Borsuk-Ulam theorems proved by Dold with methods using the Vietoris mapping theorem we show that Dold's results can be extended to multivalued maps. Such methods were invented by Eilenberg and Montgomery who applied them to multivalued fixed-point theorems, and they were used by Jaworowski to prove a multivalued version of the BorsukUlam theorem. Subsequently they were extended and refined in various ways by Górniewicz and others. We also indicate how our results can be proved in the relative case, for pairs of spaces rather than for single spaces only. This allows us to obtain positive results for bundles over manifolds with boundary; for instance, over a closed interval.
\end{abstract}

\section{INTRODUCTION}

Let $\pi: E \rightarrow B, \pi^{\prime}: E^{\prime} \rightarrow B$ be vector bundles over the same space $B$. Let $S E \subset E$ be the sphere bundle of $E$ and let $f: S E \rightarrow E^{\prime}$ be a fibre preserving $\left(\pi^{\prime} f=\pi\right)$ map. Parametrized Borsuk-Ulam theorems (for the standard antipodal action of the group $\mathbb{Z}_{2}$ ) are concerned with describing the size of the set $Z_{f}=\{x \in S E \mid f(x)=f(-x)\}$. Such studies were initiated by Jaworowski $[\mathrm{J} 2, \mathrm{~J} 3]$ and later developed and extended by Nakaoka [N], Fadell and Husseini [FH], Izydorek [I], and Dold [D].

In this paper we show that Dold's results are valid also for multivalued (admissible) maps in the following sense.

1.1. Definition. Let $X$ and $Y$ be spaces and let $f$ be a multivalued map from $X$ to $Y$, i.e., a function that assigns to each $x \in X$ a nonempty subset $f(x)$ of $Y$. We say that $f$ is upper semicontinuous if each $f(x)$ is compact and if the following condition holds: For every open subset $V$ of $Y$ containing $f(x)$ there exists an open subset $U$ of $X$ containing $x$ such that for each $x^{\prime} \in U$, $f\left(x^{\prime}\right) \subset V$.

For instance, if $X$ and $Y$ are compact then $f$ is upper semicontinuous iff its graph is closed in $X \times Y$.

1.2. Definition. A multivalued map $f$ from $X$ to $Y$ is said to be $\mathbb{Z}_{2}$-admissi-

Received by the editors January 27, 1991.

1991 Mathematics Subject Classification. Primary 55M20, 55R25; Secondary 54C60. 
ble (briefly, admissible) if there exists a space $\Gamma$ and two single-valued continuous maps $\alpha: \Gamma \rightarrow X$ and $\beta: \Gamma \rightarrow Y$ such that

(i) $\alpha$ is a Vietoris map, i.e., it is surjective, proper, and each set $\alpha^{-1}(x)$ is $\mathbb{Z}_{2}$-acyclic.

(ii) For each $x \in X$ the set $\beta\left(\alpha^{-1}(x)\right)$ is contained in $f(x)$.

We will say that the pair $(\alpha, \beta)$ is a "selected pair" for $f$.

For instance, if each $f(x)$ is acyclic (and if $f$ is upper semicontinuous) then $f$ is admissible.

Throughout this paper we assume that the spaces considered here are paracompact. We use the Čech cohomology theory $H^{*}$ with coefficients mod 2 (the coefficient group $\mathbb{Z}_{2}$ will be suppressed from the notation).

Let $\pi: E \rightarrow B, \pi^{\prime}: E^{\prime} \rightarrow B$ be vector bundles with the sphere bundle $S E \subset$ $E$ as above, and let $f$ be an admissible multivalued map from $S E$ to $E^{\prime}$ that is fibre-preserving ( $\pi^{\prime} \circ f=\pi$, i.e., for each $x \in S E, f(x)$ is contained in the fibre $\left.\pi^{\prime-1}(\pi x)\right)$. Let $Z_{f}=\{x \in S E \mid f(x) \cap f(-x) \neq \varnothing\}$.

By identifying antipodal points in $S E$ we obtain the projective bundle $\bar{\pi}: \bar{S} E$ $\rightarrow B$ of $E$ and 2-sheeted coverings $S E \rightarrow \bar{S} E$ and $Z_{f} \rightarrow \bar{Z}_{f}$; let $u \in H^{1} \bar{S} E$ and $u_{f} \in H^{1} \bar{Z}_{f}$ be their characteristic classes. Now we can proceed as in [D]: Let $H^{*} B[t]$ be the polynomial ring over $H^{*} B$ in one indeterminate $t$. Let $m$ and $n$ be the fibre dimensions of $E$ and $E^{\prime}$, respectively. We use Stiefel-Whitney classes $w_{j} E, w_{j} E^{\prime} \in H^{j} B$ and Stiefel-Whitney polynomials $\sum_{j=0}^{m}\left(w_{j} E\right) t^{m-j}$ and $\sum_{j=0}^{n}\left(w_{j} E^{\prime}\right) t^{n-j}$. Since $H^{*} \bar{S} E$ and $H^{*} \bar{Z}_{f}$ are $H^{*} B$ algebras (via $\bar{\pi}^{*}: H^{*} B \rightarrow H^{*} \bar{S} E$ ), we can substitute $u$ and $u_{f}$ for the indeterminate $t$ and obtain a homomorphism of $H^{*} B$-algebras

$$
\sigma: H^{*} B[t] \rightarrow H^{*} \bar{S} E \rightarrow H^{*} \bar{Z}_{f}, \quad t \rightarrow u .
$$

\section{Main Results}

2.1. Theorem. If $q(t) \in H^{*} B[t]$ is such that $q\left(u_{f}\right)=0$ then $q(t) w^{\prime}(t)=$ $w(t) q^{\prime}(t)$ for some polynomial $q^{\prime}(t) \in H^{*} B[t]$.

2.2. Corollary. If $m, n$ are the fibre dimensions of $E, E^{\prime}$, respectively, then $q\left(u_{f}\right) \neq 0$ for all polynomials $q(t)$ whose degree with respect to $t$ is smaller than $m-n$. In other words, the $H^{*} B$-homomorphism

$$
\bigoplus_{i=0}^{m-n-1}\left(H^{*} B\right) t^{i} \rightarrow H^{*} \bar{Z}_{f}, \quad t^{i} \rightarrow u_{f}^{i}
$$

is monomorphic. In particular, if $m>n$ then $\operatorname{cohom} \operatorname{dim}\left(\bar{Z}_{f}\right) \geq \operatorname{cohom} \operatorname{dim}(B)$ $+m-n-1$, where cohom dim denotes the cohomological dimension.

As a special case of Theorem 2.1 we obtain the following theorem, which was proved in [J2] for single-valued maps.

2.3. Corollary. Let $\pi: S \rightarrow B$ be an $n$-sphere bundle with the antipodal involution, let $\pi^{\prime}: E^{\prime} \rightarrow B$ be an $\mathbb{R}^{k}$-bundle, and let $f$ be an admissible multivalued fibre preserving map from $S$ to $E^{\prime}$ over $B$. Then there is an injective map $H^{j} B \rightarrow H^{j+n-k} \bar{Z}_{f}$. In particular, if $k=n$ then this is map induced by the projection $\bar{Z}_{f} \rightarrow B$; i.e., $\left(\bar{\pi} \mid \bar{Z}_{f}\right)^{*}: H^{j} B \rightarrow H^{j} \bar{Z}_{f}$ is injective for all $j \geq 0$. 
2.4. Corollary. If $B$ is a closed manifold and $f$ is an admissible multivalued fibre preserving map of an $n$-sphere bundle $\pi: S \rightarrow B$ with the antipodal involution to an $\mathbb{R}^{k}$-bundle $\pi^{\prime}: E^{\prime} \rightarrow B$ then $\operatorname{dim} \bar{Z}_{f} \geq \operatorname{dim} B+n-k$, where $\operatorname{dim}$ denotes the covering dimension.

\section{Proof of Theorem 2.1}

We will first prove a lemma that is a version of Theorem (1.3) from [D] adapted to our situation. We show that Dold's theorem is valid not just for maps $f: S E \rightarrow E^{\prime}$, but also in the following, more general setting.

Suppose that $X$ is any space with a free involution $a: X \rightarrow X$ and $v: X \rightarrow$ $S E$ is an equivariant Vietoris map. Let $g: X \rightarrow E^{\prime}$ be a single-valued map that makes the diagram

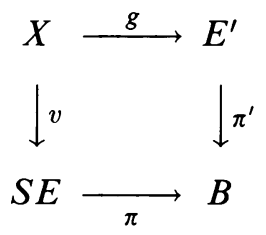

commutative, $\pi^{\prime} g=\pi v$. Set $X_{g}=\{x \in X \mid g x=g(a x)\}$.

Just as $H^{*} \bar{S} E$ and $H^{*} \bar{Z}_{f}$ (see the introduction) were $H^{*} B$-algebras via $\bar{\pi}^{*}: H^{*} B \rightarrow H^{*} \bar{S} E, H^{*} \bar{X}$ and $H^{*} \bar{X}_{g}$ are $H^{*} B$-algebras via the homomorphism $\bar{v}^{*} \circ \bar{\pi}^{*}: H^{*} B \rightarrow H^{*} \bar{X}$. Thus the characteristic class $u_{g} \in H^{1} \bar{X}_{g}$ of the involution $a \mid X_{g}: X_{g} \rightarrow X_{g}$ can be substituted for $t$ to any polynomial $q(t) \in H^{*} B[t]$.

Theorem (1.3) of [D] corresponds to the following lemma in our setting.

3.1. Lemma. If $q(t) \in H^{*} B[t]$ vanishes on $\overline{X_{g}}, q\left(u_{g}\right)=0$, then there is a polynomial $q^{\prime}(t) \in H^{*} B[t]$ such that $q(t) w^{\prime}(t)=w(t) q^{\prime}(t)$.

Proof. It is well known (and easily seen) that if $X$ and $Y$ are free $\mathbb{Z}_{2}$-spaces and $X \rightarrow Y$ is an equivariant Vietoris map then the induced map $\bar{X} \rightarrow \bar{Y}$ of the orbit spaces is also a Vietoris map. Thus, in our case, $\bar{v}: \bar{X} \rightarrow \bar{S} E$ is a Vietoris map.

We will not repeat Dold's argument step by step; we will only note that his proof can be adapted to our setting due to the fact that the homomorphism $\bar{v}^{*}$ induced by the Vietoris map $\bar{v}$ is an isomorphism. Thus, as far as the cohomology is concerned, the arrows $S E \leftarrow X \rightarrow E^{\prime}$ work just as well as a single arrow $S E \rightarrow E^{\prime}$.

Proof of 2.1. Given an admissible multivalued map $f$ from $S E$ to $E^{\prime}$, choose a space $\Gamma$ and (single-valued) maps $\alpha$ and $\beta$ such that $(\alpha, \beta)$ is a "selected pair" for $f$ (see Definition 1.2). Let $X=\left\{\left(\gamma, \gamma^{\prime}\right) \in \Gamma \times \Gamma \mid \alpha(\gamma)=-\alpha\left(\gamma^{\prime}\right)\right\}$. Consider the following commutative diagram

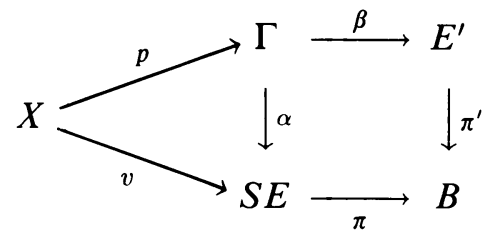


Here $p$ is the first projection, $\left(\gamma, \gamma^{\prime}\right) \rightarrow \gamma$, and $v=\alpha \circ p$. Then $v$ is a Vietoris map since for each $x \in S E, v^{-1}(x)=\alpha^{-1}(x) \times \alpha^{-1}(-x)$ and $\alpha^{-1}(x)$ and $\alpha^{-1}(-x)$ are acyclic.

The space $X$ admits a free involution $\left(\gamma, \gamma^{\prime}\right) \rightarrow\left(\gamma^{\prime}, \gamma\right)$ and $v: X \rightarrow S E$ becomes then an equivariant map. Let $g=\beta \circ p: X \rightarrow E^{\prime}$. Notice that if $h\left(\gamma, \gamma^{\prime}\right)=h\left(\gamma^{\prime}, \gamma\right)$ for some $\left(\gamma, \gamma^{\prime}\right) \in X$ then $f(\alpha(\gamma)) \cap f\left(\alpha\left(\gamma^{\prime}\right)\right) \neq \varnothing$. Thus $v\left(X_{g}\right) \subset A_{f}$ and by the naturality of characteristic classes with respect to equivariant maps,

$$
\left(\bar{v} \mid \overline{X_{g}}\right)^{*}\left(u_{f}\right)=u_{g} \in H^{1} \overline{X_{g}} .
$$

Thus $q\left(u_{g}\right)=q\left(\left(\bar{v} \mid \overline{X_{g}}\right)^{*}\left(u_{f}\right)\right)=\left(\bar{v} \mid \overline{X_{g}}\right)^{*}\left(q\left(u_{f}\right)\right)=0$.

\section{Comments on the Relative CASE}

Sometimes it is useful to have relative versions of the above results. Thus (continuing with the notation used in $\S 1$ ) suppose that $B_{0}$ is a closed subset of $B, S E_{0}=\pi^{-1} B_{0}, Z_{0 f}=Z_{f} \cap S E_{0}$, and $\bar{Z}_{0 f}=\bar{Z}_{f} \cap \bar{S} E_{0}$. We work with the polynomial ring $H^{*} B[t]$. Then substitution of $u \in H^{1} \bar{S} E$ for $t$ yields a homomorphism of $H^{*}\left(B, B_{0}\right)$-algebras

$$
\sigma: H^{*} B[t] \rightarrow H^{*} \bar{S} E \rightarrow H^{*}\left(\bar{Z}_{f}, \bar{Z}_{0 f}\right) .
$$

The results of $[D, \S 1]$ remain valid in this relative case. Consequently, we also obtain relative versions of the results of $\S 2$ of this paper.

4.1. Theorem. If $q(t) \in H^{*}\left(B, B_{0}\right)[t]$ is such that $q\left(u_{f}\right)=0$ then $q(t) w^{\prime}(t)=$ $w(t) q^{\prime}(t)$ for some polynomial $q^{\prime}(t) \in H^{*}\left(B, B_{0}\right)[t]$.

4.2. Corollary. If $m, n$ are the fibre dimensions of $E, E^{\prime}$, respectively, then $q\left(u_{f}\right) \neq 0$ for all polynomials $q(t)$ whose degree with respect to $t$ is smaller than $m-n$. In other words, the $H^{*}\left(B, B_{0}\right)$-homomorphism

$$
\bigoplus_{i=0}^{m-n-1} H^{*}\left(B, B_{0}\right) t^{i} \rightarrow H^{*}\left(\bar{Z}_{f}, \bar{Z}_{0 f}\right), \quad t^{i} \rightarrow u_{f}^{i},
$$

is monomorphic. In particular, if $m>n$ then

$$
\text { cohom. } \operatorname{dim} .\left(\bar{Z}_{f}\right) \geq \text { cohom. } \operatorname{dim} .(B)+m-n-1,
$$

where cohom. dim. denotes the covering dimension.

4.3. Corollary. Let $\pi:\left(S, S_{0}\right) \rightarrow\left(B, B_{0}\right)$ be an $n$-sphere bundle over $\left(B, B_{0}\right)$ with the antipodal involution, let $\pi^{\prime}:\left(E^{\prime}, E_{0}^{\prime}\right) \rightarrow\left(B, B_{0}\right)$ be an $\mathbb{R}^{k}$-bundle, and let $f$ be an admissible multivalued fibre preserving map from $S$ to $E^{\prime}$ over $\left(B, B_{0}\right)$. Then there is an injective map

$$
H^{j}\left(B, B_{0}\right) \rightarrow H^{j+n-k}\left(\bar{Z}_{f}, \bar{Z}_{0 f}\right) .
$$

In particular, if $k=n$ this is map induced by the projection $\bar{Z}_{f} \rightarrow B$; i.e.,

$$
\left(\bar{\pi} \mid\left(\bar{Z}_{f}, \bar{Z}_{0 f}\right)\right)^{*}: H^{j}\left(B, B_{0}\right) \rightarrow H^{j}\left(\bar{Z}_{f}, \bar{Z}_{0 f}\right)
$$

is injective for all $j \geq 0$.

4.4. Corollary. If $B$ is a closed manifold with boundary $B_{0}$ and $f$ is an admissible multivalued fibre preserving map of an $n$-sphere bundle $\pi:\left(S, S_{0}\right) \rightarrow$ 
$\left(B, B_{0}\right)$, with the antipodal involution to an $\mathbb{R}^{k}$-bundle $\pi^{\prime}:\left(E^{\prime}, E_{0}^{\prime}\right) \rightarrow\left(B, B_{0}\right)$, then $\operatorname{dim} \bar{Z}_{f} \geq \operatorname{dim} B+n-k$.

If $B$ is an interval $0 \leq s \leq 1$, then this implies that there exists a continuum $C \subset Z_{f}$ joining $\pi^{-1}(0)$ with $\pi^{-1}(1)$

Remark 4.5. When using the Eilenberg-Montgomery technique [EM] for multivalued mappings, some multivalued mapping theorems may be reduced to the corresponding single-valued cases. Generally speaking, this is the case when the multivalued map in question has a single-valued cross section (or a "selector"). To have an example showing the significance of a generalization to multivalued maps, one should construct a map with acyclic (for instance, convex) values that would not be "reducible" to a single-valued map, such as that of a multivalued map without a single valued cross section (or a "selector"). Examples of this kind can easily be given; in fact, they exist already in the classical case of the Borsuk-Ulam theorem, for single spaces over a point (rather than for bundles of spaces). The example given below will be that of a (multivalued) map of $S^{1}$ into $\mathbb{R}$.

Example. Let $S^{1}$ be the unit circle on the $\left(x_{1}, x_{2}\right)$. Define a (multivalued) map $f$ from $S^{1}$ to $\mathbb{R}$ as follows:

(1) If $x_{2} \geq 0$ then $f$ is the projection $\left(x_{1}, x_{2}\right) \mapsto x_{1}$.

(2.a) If $x_{2} \leq 0$ and $x_{1}<0$ then $f\left(x_{1}, x_{2}\right)=-1$.

(2.b) If $x_{2} \leq 0$ and $x_{1}>0$ then $f\left(x_{1}, x_{2}\right)=1$.

(2.c) If $x_{2} \leq 0$ and $x_{1}=0$ (hence $=-1$ ) then $f\left(x_{1}, x_{2}\right)=[-1,1]$ (the closed interval).

One sees easily that $f$ is defined in a consistent way and is upper semicontinuous (its graph is closed). The map is admissible; its values are acyclic (even. convex): they are all single points except of one value that is a closed interval. In this case the conclusion of the (multivalued) Borsuk-Ulam theorem is, of course, valid; but that conclusion cannot be obtained directly from Dold's results because the map has no single-valued cross section.

\section{REFERENCES}

[D] A. Dold, Parametrized Borsuk-Ulam theorems, Comment. Math. Helv. 63 (1988), 275-285.

[EM] S. Eilenberg and D. Montgomery, Fixed point theorems for multi-valued transformation, Amer. J. Math. 58 (1946), 214-222.

[FH] E. R. Fadell and S. Y. Husseini, Cohomological index theory with applications to critical point theory and Borsuk-Ulam theorems, Sém. Math. Sup., vol. 108, Université de Montréal, Montréal, 1989, pp. 10-54.

[G] L. Górniewicz, Homological methods in fixed point theory for multivalued maps, Dissertationes Math. 129 (1976), 1-71.

[I] M. Izydorek, Remarks on Borsuk-Ulam Theorem for multi-valued maps, Bull. Acad. Sci. Pol. Math. 35 (1987), 501-504.

[J1] J. Jaworowski, Theorem on antipodes for multi-valued mappings and a fixed point theorem, Bull. Acad. Sci. Pol. Math. 4 (1956), 187-192.

[J2] _ A continuous version of the Borsuk-Ulam Theorem, Proc. Amer. Math. Soc. 82 (1981), $112-114$. 
[J3] _ Fibre preserving maps of sphere bundles into vector space bundles, Proceedings of the Fixed Point Theory Conference, Sherbrooke, Québec 1980, Lecture Notes in Math., vol. 886, Springer-Verlag, Heidelberg, 1981, pp. 154-162.

[N] M. Nakaoka, Equivariant point theorems for fibre-preserving maps, Osaka J. Math. 21 (1984), 809-815.

Department of Mathematics, Technical University of Gdańsk, 80-952 Gdańsk, Ul. MajaKowsKiego $11 / 12$, Poland

Department of Mathematics, Indiana University, Bloomington, Indiana 47405

E-mail address: jaworows@iubacs 